\title{
On the dynamics of meteoroid streams
}

\author{
P. Jenniskens \\ NASA/Ames Research Center, Mail Stop 239-4, Moffett Field, CA 94035-1000, U.S.A. \\ (Received January 5, 1998; Revised March 12, 1998; Accepted March 13, 1998)
}

\begin{abstract}
Zodiacal dust evolves from cometary debris through a stage called a meteoroid stream. Meteoroid streams produce meteor showers if a node of the stream is near 1 AU. On occasion, Earth encounters a stream of meteoroids that has not dispersed wide enough to be detected annually. A rare and often short lived enhancement of rates is observed during which the meteors typically have smaller radiant dispersion and sometimes anomalous fragmentation properties and end heights. Here, we summarize recent observations of these meteor outbursts and discuss how the results constrain our knowledge of the early stages of meteoroid stream formation. These stages tie meteoroid streams to cometary dust trails and are an important step in the dynamical evolution from cometary to zodiacal dust.
\end{abstract}

\section{Introduction}

The zodiacal cloud contains grains that originated from submm-cm sized debris of comets. The percentage of cometary matter is estimated variably between 10 and 70 percent at $1 \mathrm{AU}$ and close to 100 percent at heliocentric distances beyond the asteroid belt, where interstellar and Kuiper Belt impact erosion dust add mainly to the smaller grain sizes (Dermott et al., 1994; Mann, 1996). A few comets can dominate the supply of larger debris to the rapidly evolving zodiacal cloud. Initially, that debris will be in the form of a meteoroid stream, a highly non-homogeneous spatial distribution that can persist over relatively long timescales. Hence, an understanding of meteoroid stream dynamics is necessary to understand the formation history, the fraction of cometary dust to all of zodiacal dust, and the present degree of non-homogeneity of the zodiacal cloud.

A shower of meteors is observed at times when Earth encounters such a meteoroid stream. Grains that are larger than about 100 micron, and up to tens of $\mathrm{cm}$ in size, can be detected by radar and optical techniques. The variation of meteoroid flux in the Earth's path can be measured accurately as a function of meteor luminosity and the orbit of individual meteoroids can be calculated. Meteors represent the peak of the cometary mass loss curve for dust. Thus, meteor showers can be used to probe the mass loss of comets and the dynamics of meteoroid streams, albeit that only a few streams are assessable (those with a node near $1 \mathrm{AU}$ ) and then only under specific encounter conditions (Steel, 1994).

A large number of mechanisms have been proposed to explain the observed distribution of dust in the path of the Earth (see reviews in Lovell (1954), Lewin (1961), Williams (1993, 1996a), and Steel (1994)). Surprisingly few of these are substantiated by observations. Best observed are the annual showers. On top of the annual showers are the occasional meteor outbursts. Meteor outbursts are due to relatively recent ejecta that have not dispersed wide enough

Copy right (C) The Society of Geomagnetism and Earth, Planetary and Space Sciences (SGEPSS); The Seismological Society of Japan; The Volcanological Society of Japan; The Geodetic Society of Japan; The Japanese Society for Planetary Sciences. to be encountered each year. These ejecta are thought to be related to IRAS dust trails (Kresák, 1993; Jenniskens, 1995a). While the cause and nature of annual showers are determined mainly by gravitational perturbations, outbursts can still carry information about the cometary ejection process.

Until recently, meteor outbursts have eluded systematic observations. With some rare exceptions such as the outstanding study of the 1946 Draconid storm from single station photographic observations by Jacchia et al. (1950), meteor outbursts have been known mainly from sparse visual observations and the occasional detection by radar (e.g. Porubcan, 1974; Watanabe et al., 1992; Simek, 1994). Early observations are discussed in Lewin (1961) and Kresák (1980), while Jenniskens (1995a) has recalibrated the meteor counts to a common activity scale. These observations provide some information about the rate profile and the magnitude distribution index, but give few clues about the relative importance of planetary perturbations and the ejection process for example.

Beginning in 1993, a series of successful measurements of meteor outbursts were made in dedicated observations using multi-station photography, visual observations and radio forward meteor-scatter techniques. The observations followed a series of outbursts of the Perseid and Leonid showers. With a current rate of 3-5 events per year, meteor outbursts are frequent enough to be a topic of dedicated study. In addition, advances in computer technology have generated new interest in numerical models of meteoroid stream dynamics in recent years. These models offer an alternative means of testing the various proposed mechanisms. In combination with observational results, they rapidly have lead to new insight.

This paper reviews those recent developments. An attempt is made to emphasize what are the open questions in understanding the three-dimensional distribution of meteoroids in meteoroid streams and in understanding the dynamical evolution from meteoroid streams into zodiacal dust.

The paper is ordered as follows. First, a distinction between meteor outbursts from long and short period comets is made 


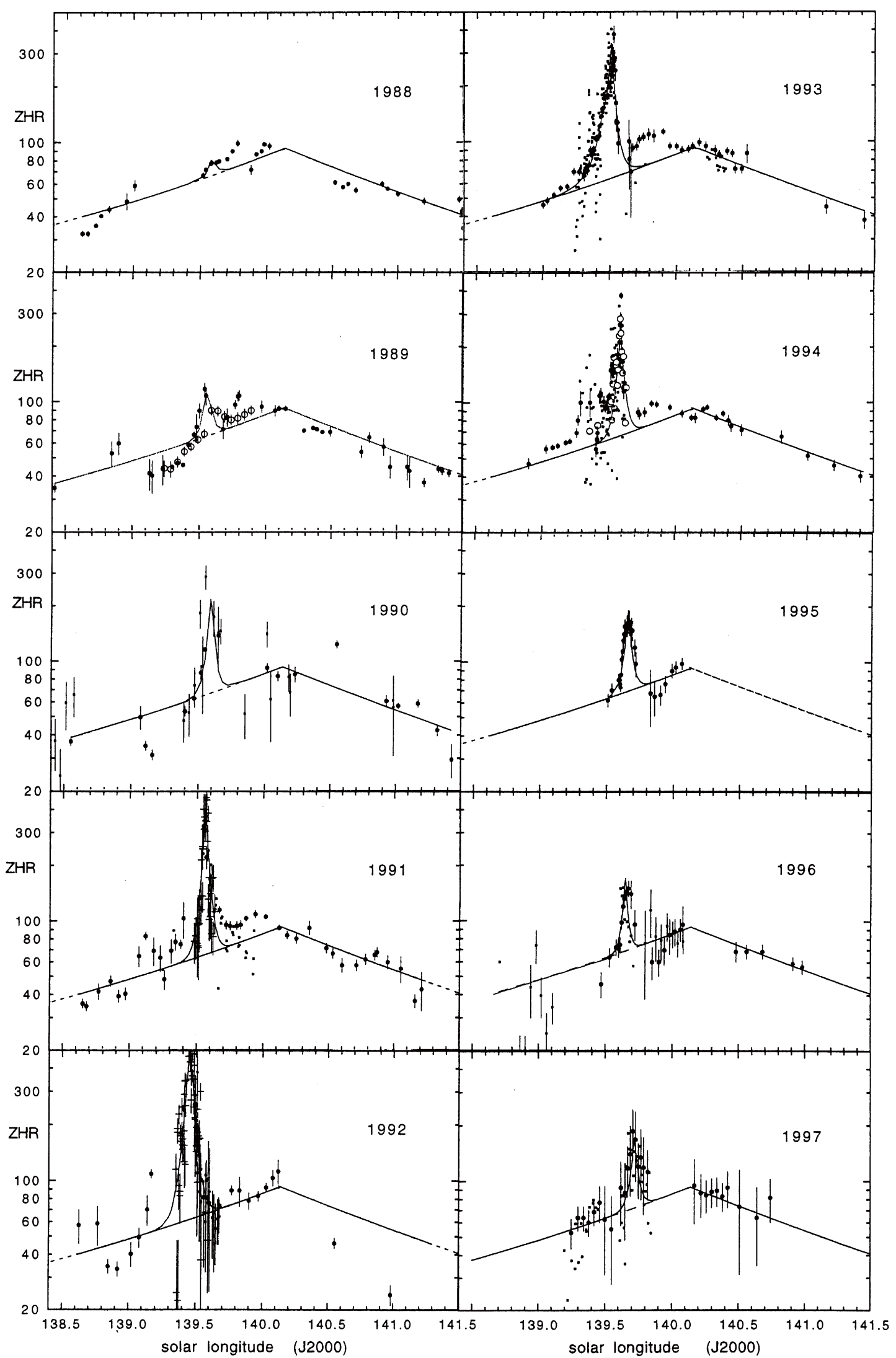

Fig. 1. Meteoroid stream activity curves of the Perseids in the years from 1988 until 1997. Note the Perseid Filament on top of the normal annual shower activity. Dots are visual observations, while crosses are radio meteor scatter observations. 
(Section 2). Subsequent chapters discuss the dispersion perpendicular to the plane of the comet orbit (Section 3), along the orbit of the comet (Section 4), and in the plane of the comet orbit (Section 5). Section 6 deals with changes in particle density, morphology, and size distribution between outburst and annual stream components. Section 7 discusses the transition from outburst to annual stream, while Section 8 is about the transition from the annual stream into a sporadic meteor background.

\section{Meteoroid Streams from Short-Period and Long-Period Comets}

Early on, it was recognized that there is a distinction between meteor outbursts that coincide with the return of the comet to perihelion (near-comet type-Jenniskens, 1995a) and those that occur in an unrelated pattern, usually when the comet is far from the Sun (far-comet type). Only recently has it become clear that near-comet type outbursts are related to comets with a short orbital period $(P=3-20 \mathrm{yr}$.) and Halley-type comets ( $P=20-200 \mathrm{yr}$.), while far-comet type outbursts are due to the debris of long-period comets (Jenniskens et al., 1997a).

An example of near-comet type outbursts are those of the Perseid shower, shown in Fig. 1. The Perseids are caused by debris of comet 109P/Swift-Tuttle, a Halley-type comet with a 135 year orbital period. The comet returned to perihelion in December of 1992. Between 1989 and the present, a brief increase of rates was observed with a characteristic duration (Jenniskens et al., 1998). Visual observations of Perseid outbursts from around the globe have been summarized by Brown and Rendtel (1996), while radar observations are given by Simek and Pecina (1996). The meteor outburst stood out above the strong annual activity of the Perseid shower, which was unaffected. Apparently, Earth encountered a distinct sheet or filament of dust, called the "Perseid Filament", confined to a small part of the orbit near the position of the comet. A similar sheet of dust was crossed during the recent Leonid outbursts in conjunction with the February 1998 return of comet 55P/ Tempel-Tuttle. Leonid outbursts were first detected in 1994 (Jenniskens, 1996a, b).

Example of far-comet type outbursts are those of the alpha-Monocerotid shower in November of 1995 (Rendtel et al., 1996; Jenniskens et al., 1997a) and the Lyrids and Aurigids in prior years. The lather are associated with longperiod comets $\mathrm{C} /$ Thatcher and $\mathrm{C} /$ Kiess respectively. Farcomet type outbursts typically occur only in one year, followed by many dormant years with only weak annual stream activity. The outbursts do not correlate with the return of the parent comet to perihelion.

\section{The Dispersion Perpendicular to the Comet Orbital Plane}

The width of those sheets, ribbons, filaments, or trails is thought to reflect the process of ejection and the ratio of radiation over gravitational forces $(\beta)$ of the meteoroids. Outstanding problems are the magnitude of these ejection velocities (Subsection 3.1), and the influence of radiation pressure and gravitational perturbations on the duration of the shower (Subsection 3.2).

\subsection{The ejection velocities}

The ejection process was first discussed by Whipple (1951), who considered the ejection of large (>10 micron) meteoroids by the gas dynamic drag of water vapor. Later studies included the effects of non-spherical grains and fluffy aggregate morphology (Gustafson, 1989a), and cooling by the sublimation of the cometary ice and the adiabatic expansion of the escaping gases (Jones, 1995; Jones and Brown, 1997). The Whipple formula is still commonly used, albeit with slightly different prefactors and exponents. For example, Jones and Brown (1997) proposed the approximate equation:

$$
\begin{aligned}
V_{\mathrm{ej}}(\mathrm{m} / \mathrm{s})= & 32.3 \mathrm{R}_{\mathrm{c}}{ }^{1 / 2}(\mathrm{~km}) m^{-1 / 6}(\mathrm{~kg}) \\
\cdot & \rho^{-1 / 3}\left(\mathrm{~kg} / \mathrm{m}^{3}\right) r^{-1.038}(\mathrm{AU}) .
\end{aligned}
$$

Most dust ejection occurs near perihelion. For a typical comet nucleus radius such as that of Leonid comet $55 \mathrm{P} /$ Tempel-Tuttle $\left(\mathrm{R}_{\mathrm{c}}=1.8 \mathrm{~km}\right.$, Hainaut et al., 1998), an $1.8 \cdot 10^{-4} \mathrm{~kg}$ particle of density $\rho=300 \mathrm{~kg} / \mathrm{m}^{3}$ (Novikov et al., 1996) ejected at $r=q=0.977$ AU would have a terminal speed of $V_{\text {ej }}=28 \mathrm{~m} / \mathrm{s}$, and would produce a -1 magnitude Leonid upon colliding with Earth. Whipple's equation was used by Harmon et al. (1997), amongst others, who modeled the radar reflection off the coma of comet C/Hyakutake, with $\mathrm{R}_{\mathrm{c}}=1.2 \mathrm{~km}$, with a velocity scaling with grain radius $V_{\mathrm{ej}} \sim \sqrt{a^{-1}}$ and $V_{\mathrm{ej}}=40 \mathrm{~m} / \mathrm{s}$ for $a=1 \mathrm{~cm}$, which translates to $V_{\mathrm{ej}}=25 \mathrm{~m} / \mathrm{s}$ for a Leonid of magnitude -1 . In another example, Chifo (1997) discussed sublimation of dusty ices and derived ejection velocities translating to $15-37 \mathrm{~m} / \mathrm{s}$ for a -1 Leonid.

Direct observations of ejection velocities have thus far been possible only for the smallest grains that are seen in scattered sunlight and make up comet jets and arcs. C/Hale Bopp's jets, for example, have been observed to expand at about $250-300 \mathrm{~m} / \mathrm{s}$, and are thought to consist of particles with a value of $\beta \sim 0.1-1$. From Eq. (1), it follows that $V_{\mathrm{ej}}$ should scale according to:

$$
V_{\mathrm{ej}} \sim(0.3-1) \cdot 10^{3} \sqrt{\beta} \mathrm{m} / \mathrm{s} .
$$

Unfortunately, the relationship between $\beta$ and mass (and meteor magnitude) remains uncertain, mainly because of an uncertain dust density that may vary as a function of particle mass. If we assume that a -1 magnitude Leonid corresponds to $\beta=3 \cdot 10^{-4}$, then this simple scaling suggests an ejection velocity of $V_{\mathrm{ej}}=5-17 \mathrm{~m} / \mathrm{s}$ if ejected from C/Hale-Bopp and $V_{\text {ej }}=24-80 \mathrm{~m} / \mathrm{s}$ in the case of the smaller comet $55 \mathrm{P} /$ Tempel-Tuttle (because $V_{\mathrm{ej}} \sim \mathrm{R}_{\mathrm{c}}{ }^{1 / 2}$ according to Eq. (1)).

In contrast, ejection velocities of $1-10 \mathrm{~m} / \mathrm{s}$ have been derived from models describing the thermal infrared emission of cometary dust trails (Sykes and Walker, 1992). Those speeds are mainly constrained by the width of the dust trails and refer to the out-of-plane ejection speed $V_{\text {ej }} \perp$, because the trails are mostly seen from within the orbital plane. If the trail width $(D)$ represents the maximum excursion of trail particles from the comet orbital plane and the Earth is in the orbital plane, then (Sykes et al., 1990): 


$$
V_{\mathrm{ej}} \perp=V_{\mathrm{c}} \cdot(0.5 D) / r
$$

with $V_{\mathrm{c}}$ the orbital velocity of the comet at the time of emission and $r$ the heliocentric distance of the trail.

Kresák (1993) pointed out that the width of the cometary dust trails are similar to the width implied by the duration of meteor storms. Indeed, because the infrared emission spectrum implies grain sizes of similar magnitude, a link between both is expected. We can now use the fact that stream width and nodal dispersion $\Delta \Omega$ are related according to (Kresák and Porubcan, 1970):

$$
D=2 r \cdot \tan (\Delta \Omega / 2) \cdot \sin \varepsilon_{\mathrm{h}}
$$

with $r$ and $D$ in AU and $\varepsilon_{\mathrm{h}}$ the angle between the radiant of the heliocentric velocity and the Earth's apex. $\varepsilon_{\mathrm{h}}=18.1$ degree for the Leonids. Leonid storms have an equivalent duration of $\Delta \Omega=0.029 \pm 0.003$ degrees (Jenniskens, 1995a). If the width of the $55 \mathrm{P} /$ Tempel-Tuttle dust trail is represented by the duration of past Leonid storms (Jenniskens, 1995a), then this equation results in $V_{\mathrm{ej}} \perp \sim 3.3 \mathrm{~m} / \mathrm{s}$, a factor of ten less than previous estimates.

Studies of meteor outbursts could in principle constrain the ejection velocities, and settle the question if ejection velocities are of order $3 \mathrm{~m} / \mathrm{s}$ or $30 \mathrm{~m} / \mathrm{s}$ for -1 magnitude Leonids, by studies of meteoroid velocity dispersions. But rather than deciding for one of these answers, even higher ejection velocities have been derived. Pittich(1991) proposed that the observed dispersion in the semi-major axis of annual meteoroid streams reflected the ejection process itself, followed by Williams (1996b) who derived velocities of order $160-880 \mathrm{~m} / \mathrm{s}$. Harris et al. (1995) derived high ejection velocities of $V_{\mathrm{ej}}=600 \mathrm{~m} / \mathrm{s}$ from the nodal dispersion of the annual Perseid shower. Wu and Williams (1996) derived a maximum ejection velocity of $830 \mathrm{~m} / \mathrm{s}$ from photographed Leonid orbits in 1966 and 1965 (Lindblad et al., 1993), and continued to calculate a Leonid stream model with mean ejection velocities in the range 130-240 m/s (Williams, private communication). That is up from other recent models, for which mean ejection values were only 44-75 m/s (Williams and Wu, 1994; Wu and Williams, 1995a; Arter and Williams, 1997).

The rationale for these high ejection velocities came from questioning the gas to dust coupling or even the mechanism of acceleration underlying Whipple's ejection model. The outflow velocity of the water vapor is of the order of the mean thermal velocity of the water molecules at a few hundred $\mathrm{K}$ plus acceleration due to the pressure gradient, amounting to approximately $950 \mathrm{~m} / \mathrm{s}$ for ejection at $1 \mathrm{AU}$ (Jones and Brown, 1997). Dust-gas coupling is strongest close to the surface, while the gas flow is thought to have little effect further out. Hence, depressed active areas on the comet nucleus can enhance the ejection velocities by exposing the grains to a brief period of rapid acceleration before leaving the pit (Jones, 1995; Jones and Brown, 1997). Gustafson (1989a) made the point that flake- and needle shaped meteoroids can attain high ejection velocities mainly because the ejection velocity is determined by their shortest particle dimension when the grains are expected to align with the gas flow. And Steel (1994) pointed out, without elaborating, that individual meteoroids may be accelerated after initial ejection by the asymmetric evaporation of water ice while being part of the extended source of gas in the comet coma, which perhaps could increase ejection velocities above $50 \mathrm{~m} / \mathrm{s}$. Evidence of icy grains in the comet coma have been reported (e.g. Harris et al., 1997).

Kresák (1992) argued strongly against the hypothesis of such high ejection velocities, pointing at the exaggeration of dispersions by measurement error. Moreover, past observations of meteor outbursts are plenty evidence of much smaller dispersion than observed in the annual shower. First of all, outbursts are typically shorter in duration, with an equivalent width of the order of $0.3-1$ hours (Jenniskens, 1995a), as opposed to 2-4 days for the annual showers (Jenniskens, 1994). This implies a small dispersion in the node of the orbits. Secondly, single-station photographic observations of the 1946 Draconid storm demonstrated a very small radiant dispersion, meaning a small dispersion in the direction of the meteoroid velocity vectors perpendicular to the plane of the sky (Jacchia et al., 1950).

Recent observations support Kresák's position. We now have succeeded in measuring the radiant and speed of individual meteoroids in multi station photographic networks operated by amateur meteor observers. Examples of radiant distributions for the Perseid, Leonid and alpha-Monocerotid outbursts are shown in Figs. 2-4 (Jenniskens et al., 1997a, 1998; Betlem et al., 1997). For the first time, we can now distinguish between the dispersion in right ascension and that in declination. Or in other words: in and out of the ecliptic plane.

Let us first consider the dispersion out of the ecliptic plane (and out of the cometary plane). Figure 2 compares the radiant positions of the precisely reduced orbits of meteoroids obtained during the Perseid outbursts in 1993 and 1994 with the much larger dispersion measured for the annual Perseids by the same technique (Jenniskens et al., 1998). The annual Perseid shower is a strong shower and must have contributed many, if not all, of the outlayers in the radiant distribution of 1993/94. Hence, the central cluster of radiants is the signature of the outburst. The outburst activity profile consisted of two components: the "Perseid Filament" of Fig. 1 plus an increase of rates near the node of the comet orbit causing an asymmetry in the outburst profile in 1993 unlike other years. This asymmetry we called the "Perseid Nodal Blanket". The smallest radiant dispersion for the Nodal Blanket was measured perpendicular to the ecliptic plane: $\triangle \mathrm{DEC} \sim \pm 0.02^{\circ}\left(<0.03^{\circ}\right)$. From this, the maximum dispersion of velocity vectors in the plane of the sky equals to first order, with $V_{\mathrm{g}}=V_{\mathrm{h}} \sin \varepsilon_{\mathrm{h}} / \sin \varepsilon_{\mathrm{g}}$ :

$$
\Delta V_{\mathrm{h}}=V_{\mathrm{g}} \cdot \tan (\Delta \mathrm{DEC}) \cdot \cos \left(\varepsilon_{\mathrm{h}}-\varepsilon_{\mathrm{g}}\right)
$$

or $\Delta V_{\mathrm{h}}<28 \mathrm{~m} / \mathrm{s}$. This is an upper limit, because the measured dispersion is accounted for by observational error. The median ejection velocity perpendicular to the orbital plane of parent comet 109P/Swift-Tuttle must have been less. The equivalent duration of the Perseid outbursts $\left(\Delta \Omega=0.058^{\circ}\right)$ implies $V_{\mathrm{ej}}=21 \mathrm{~m} / \mathrm{s}$, in good agreement. With $\mathrm{R}_{\mathrm{c}}=11.8 \mathrm{~km}$ (O'Ceallaigh et al., 1995), Eq. (1) would predict $V_{\mathrm{ej}}=68$ $\mathrm{m} / \mathrm{s}$ for a Perseid of magnitude -1 . Hence, the Perseid 


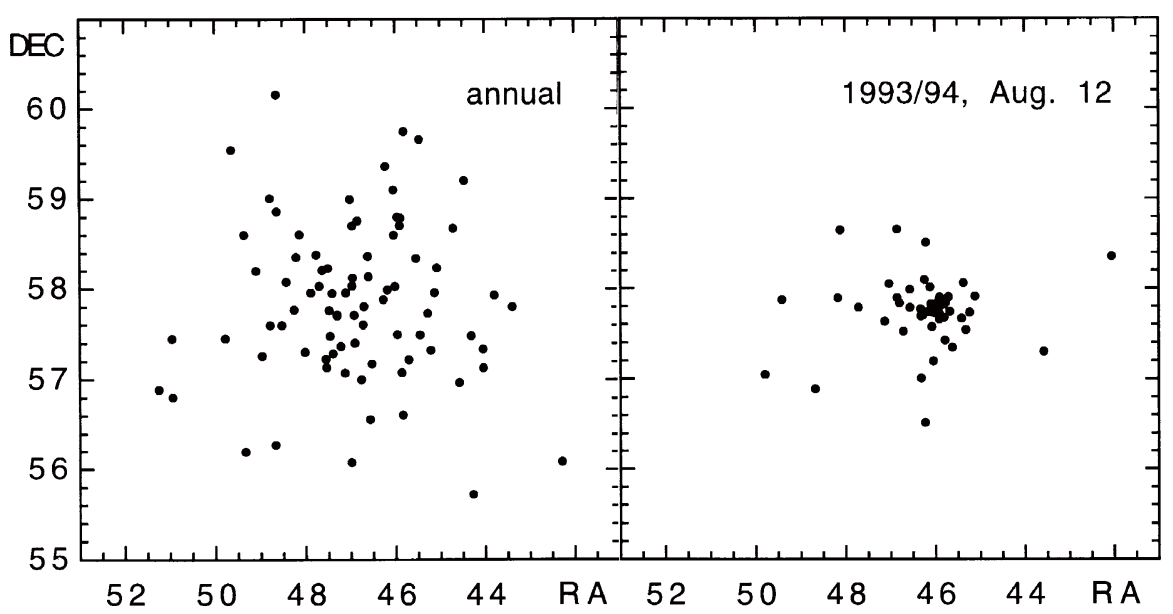

Fig. 2. Radiant dispersion of the annual and outburst Perseids (from Jenniskens et al., 1998).

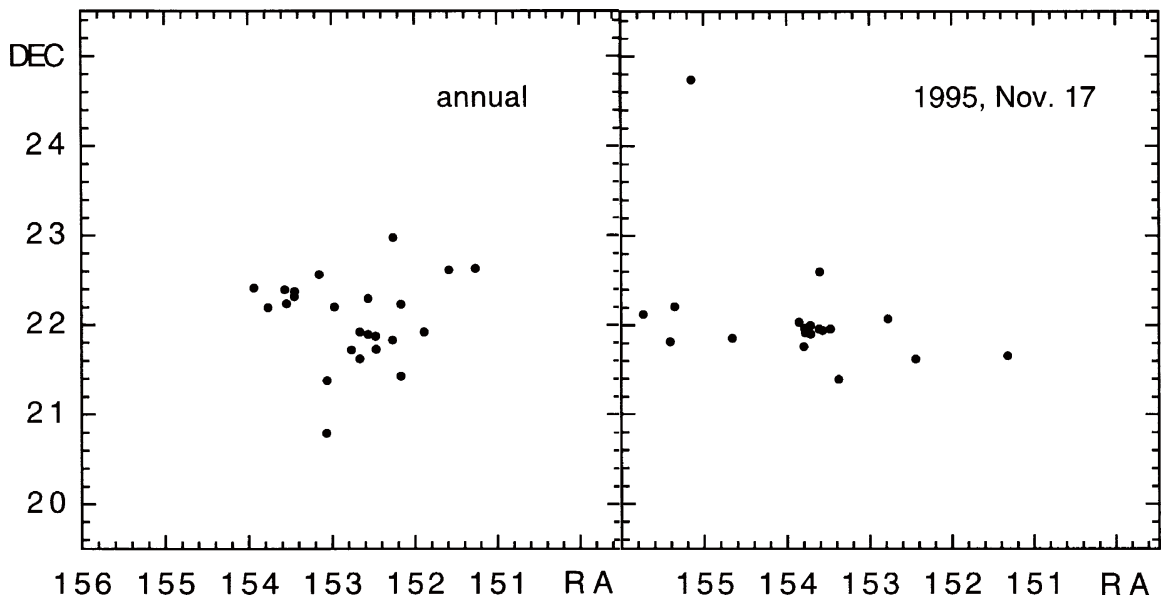

Fig. 3. As Fig. 2. The radiant dispersion during the 1995 Leonid outburst (Betlem et al., 1997) is compared with that of annual Leonids in the IAU database.

measurements suggest smaller ejection velocities than described by Eq. (1).

During the 1995 Leonid outburst, a small cluster of radiants stood out above the annual stream background (Betlem et al., 1997). The radiant dispersion of the central cluster in Fig. 3 (with only $N=9$ radiants) equals $\triangle \mathrm{DEC} \sim$ $0.08^{\circ}$, which implies a velocity component perpendicular to the 55P/Tempel-Tuttle comet orbit of less than $V_{\text {ej }}<100$ $\mathrm{m} / \mathrm{s}$, consistent with all estimates above. But clearly, the small dispersion argues against the present orbits of Leonids in the IAU database being of high precision and part of a Leonid outburst as assumed by Wu and Williams (1996). Hence, the $140-240 \mathrm{~m} / \mathrm{s}$ ejection velocities used by $\mathrm{Wu}$ and Williams and the resulting Leonid stream models are incorrect.

Finally, a narrow radiant dispersion was measured for the meteoroids that were part of the 1995 alpha-Monocerotid outburst (Fig. 4). This far-comet type outburst is caused by an unknown long-period comet. That comet is of interest because it is an impact hazard. From all our ten measured orbits, mostly obtained by video techniques, we have a

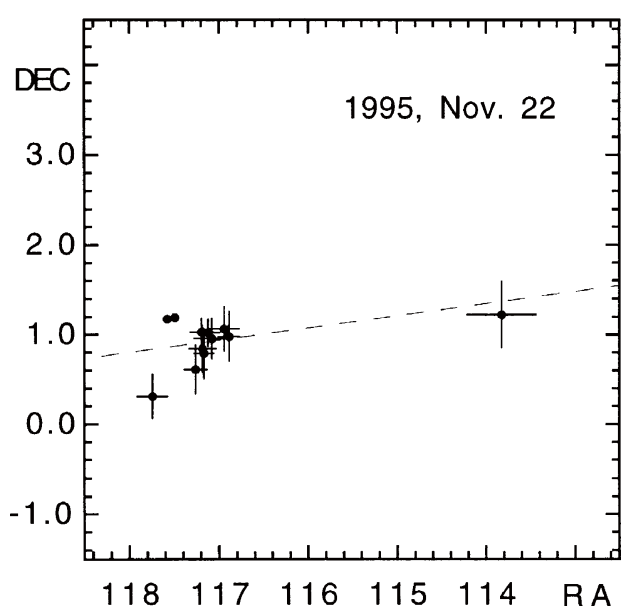

Fig. 4. As Fig. 2. The alpha-Monocerotid radiants in November 1995 (data from Jenniskens et al., 1997a-one more annual $\alpha$-Monocerotid orbit was found in the video data by de Lignie and Betlem (1997)). 
relative large dispersion of $\triangle \mathrm{DEC}=0.25^{\circ}$, but with a median observational error of $\pm 0.23^{\circ}$ per radiant, hence $V_{\text {ej }}<240$ $\mathrm{m} / \mathrm{s}$. The more accurate photographic orbits $(N=3)$ give $\Delta \mathrm{DEC}=0.096^{\circ}$ for an error of $\pm 0.1^{\circ}$, hence $V_{\mathrm{ej}}<100 \mathrm{~m} / \mathrm{s}$. The duration of the outburst $\Delta \Omega=0.011^{\circ} \pm 0.002$, implies $V_{\mathrm{ej}}=14 \mathrm{~m} / \mathrm{s}$ for a +2 magnitude alpha-Monocerotid (factor of two higher than given in Jenniskens et al. (1997a)). Equation (1) predicts $V_{\mathrm{ej}}=63 \sqrt{\mathrm{R}_{\mathrm{c}}} \mathrm{m} / \mathrm{s}$. Hence, the comet diameter must be only $0.1 \mathrm{~km}$ to be consistent with the value derived from the duration of the shower, smaller than that of typical comets. Clearly, the results from both ways of calculating the ejection velocities do not agree.

These results confirm the very small radiant dispersion inferred from single-station photographic observations during the 1946 Draconid storm (Jacchia et al., 1950). A radiant dispersion of $\pm 0.052^{\circ}$ was measured, implying $V_{\mathrm{ej}} \leq$ $14 \mathrm{~m} / \mathrm{s}$, while the duration $\Delta \Omega=0.051^{\circ}$ gives $V_{\mathrm{ej}}=9 \mathrm{~m} / \mathrm{s}$. This observation supports the very small ejection velocities.

Those that are still not convinced that meteoroid streams start out with a relatively low ejection velocity dispersion may pose the question: "Could the annual meteoroid stream hide a high- $V_{\mathrm{ej}}$ component of dust emission?" No, some increase of annual stream activity would have been expected in conjunction with the return of the Perseid and Leonid comet and none has been observed. A high-velocity component would also have increased the apparent radiant of the Draconid shower measured by Jacchia et al. (1950).

\subsection{Other influences on stream width}

The stream flux profile can be measured very accurately during an outburst for a range of meteor magnitudes. Outstanding problems are: 1) the mass-dependence of the duration of the shower; 2) the presence of filamentary structure; and 3 ) the influence of gravitational perturbations.

From Eq. (1), one would expect that the smaller particles are ejected with higher ejection velocities and are therefore spread out further. Surprisingly, the expected mass-dependence of duration: $\Delta \Omega \sim V_{\mathrm{ej}} \sim m^{-1 / 6}$ has not been observed. The +3 magnitude visually observed meteoroids should have 1.8 times broader profile than the -1 magnitude photographic meteoroids. Although the observed alpha Monocerotid flux profiles are not inconsistent with the expected larger dispersion for smaller particles (Jenniskens et al., 1997a), the Perseid outbursts do not confirm the expected behavior (Jenniskens et al., 1998).

One possibility is that the expected variation of stream width with mass is hidden by a superposition of various depositions at different epochs during slightly different relative positions of comet and meteoroid stream or for different jet activity. The discreet nature of jets almost implies some structure in the distribution of large grains, the signature of which would be filamentary structure in the stream flux profile. Although filamentary structure has been reported in some outburst flux profiles (Kresák and Slancikova, 1975; Simek, 1994), none of the reports go beyond statistical and systematic uncertainty. For a discussion see Jenniskens (1995a). The recent analysis of 1-minute counts of the 1995 alpha-Monocerotid outburst did not lead to confirmation of initial reports of a multiple maximum (Jenniskens and Docters van Leeuwen, 1997). The absence of such filamentary structure has important implications. It implies that the vertical distribution of dust homogenizes over time, either as a result of the changing position angle of the jets or as a result of planetary perturbations.

Radiant dispersions are a sensitive tool for measuring the influence of planetary perturbations. Planetary perturbations are about 30 times more effective in the direction perpendicular to the orbital plane (reflected in RA, DEC, and the node of the shower), than in the direction of motion (speed) when integrated over 10 orbital revolutions (Kresák, 1992). This is thought to be because strong perturbations occur mainly near aphelion and near the most distant node, while the change of orbital elements reflect the stability of the Tisserand invariant with respect to Jupiter. The resulting changes mostly affect the right ascension of the radiant, much less so the declination.

Indeed, some indication of this is seen in the relatively larger spread in RA found for the Perseid, Leonid and alphaMonocerotid outburst radiant dispersions (Figs. 2-4). The narrow Nodal Blanket cluster has $\triangle \mathrm{RA}= \pm 0.08^{\circ}$, while $\Delta \mathrm{DEC}$ $= \pm 0.02^{\circ}$, for example. The significant dispersion is mainly along the ecliptic plane. Future work can measure these dispersions quantitatively, which should help confine the ejection history of the meteoroids.

I conclude that planetary perturbations are important at early stages of meteoroid stream evolution and may contribute to the observed width of dust trails. That conclusion is consistent with a relatively old age for cometary dust trails, as proposed by Sykes and Walker (1992). The trails do not consist of fresh ejecta of one return only, but are the sum of 10 or so episodes of dust injection during previous returns to the inner parts of the solar system. However, note that most current models of cometary dust trails do not include such perturbations.

\section{Dispersion along the Orbit}

It is clear that the annual return of Perseid outbursts (Fig. 1) reflects a rapid dispersion of meteoroids along at least some part of the comet orbit. This is known to be a consequence of small orbital period differences resulting from the ejection velocities and the solar radiation pressure. Outstanding problems are: disagreements in 1) the expected size-dependence of dispersion, 2) the expected asymmetry before and after the position of the comet, and 3 ) the rate of dispersion. Discussion of item 3 will be postponed until Section 7.

\subsection{Dispersion in the orbit}

Plavec (1955) first recognized that Whipple's ejection model implied that particles tend to rapidly disperse along the comet orbit, because small differences in velocities near perihelion result in rather different orbital periods. Initially, the large particles do not move far into the comet coma and stay relatively close to the comet nucleus for an Earth bound observer. One orbit later, the grains will be dispersed in a trail-like structure as a result of the different orbital periods (see upper part of Fig. 5). The extend of that trail is a function of the ejection velocity relative to the parent comet's heliocentric velocity $V_{\mathrm{h}}$. The dispersion in semi-major axis $\Delta a$ from a dispersion in speed $\Delta V_{\mathrm{h}}$ follows directly from the energy integral: 


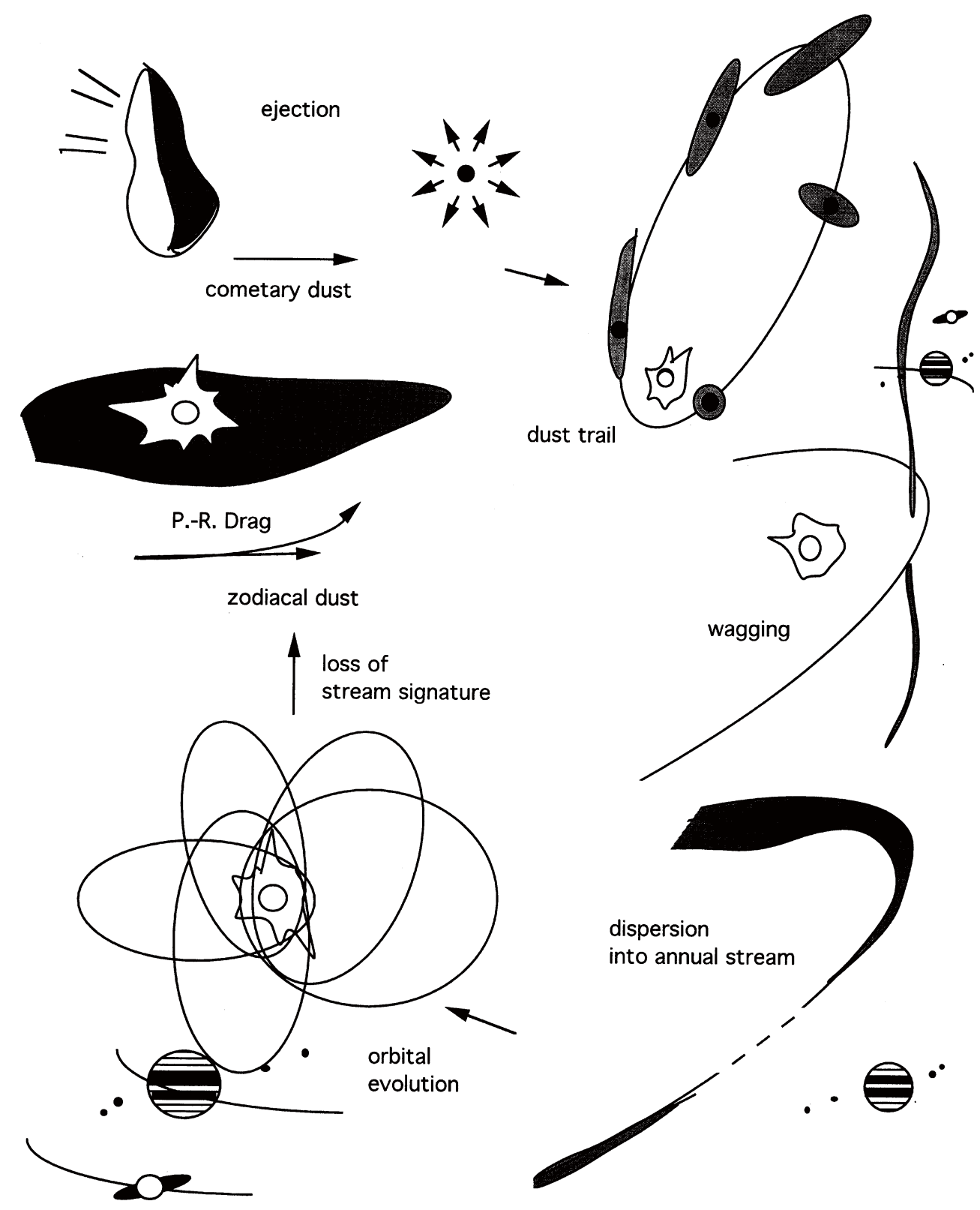

Fig. 5. A cartoon illustrating the dynamical evolution from cometary debris to zodiacal dust.

$$
V_{\mathrm{h}}=k((2 / r)-(1 / a))^{1 / 2}
$$

with $k=29.79 \mathrm{AU}^{1 / 2} \mathrm{~km} \mathrm{~s}^{-1}$. The rate of dispersion is given by (Sykes and Walker, 1992):

$$
\begin{aligned}
\Delta a= & \beta a((1+e) /(1-e)) \\
& +2 a V_{\mathrm{ej}}\left[\left(a / \mathrm{GM}_{\mathrm{o}}\right)(1+e) /(1-e)\right]^{1 / 2} .
\end{aligned}
$$

The first term describes the effect due to radiation pressure, the second due to ejection velocity vector in the direction of orbital motion. The trail length grows with successive revolutions, the grains furthest away from the comet being the oldest ejecta.

Such dust trails were first observed in the orbit of shortperiod comets by IRAS in 1983 (Davies et al., 1984; Sykes et al., 1986). The dispersion of dust before and after the comet compared favorably with the dispersion of dust as observed in the near-comet type meteor outbursts of the Perseids, Leonids and Draconids. This, again, suggests a link between cometary dust trails and meteor storms (Kresák, 1993).

\subsection{Particles lagging the comet}

Kresák (1976) first realized that the radiation pressure on the meteoroids would result in different effective gravitational fields for comet and meteoroids, causing the dust to have systematically longer orbital periods on average and causing the dust to lag the comet during subsequent returns. An asymmetry in the dust distribution would be expected, favoring the region behind the comet.

This effect seems to be confirmed for the meteoroids ejected from comet 55P/Tempel-Tuttle. Sekanina (1975) and Yeomans (1981) used changing encounter conditions during each return and the past accounts of Leonid storms to produce a map of highest dust densities. They found that 
dust densities are highest just outside of the comet orbit and behind the comet.

In other cases the situation is clearly more complex. Similar mapping of one short-period comet 21P/GiacobiniZinner (Draconids) does not confirm this trend: dust is found outside the comet orbit before the comet and inside the comet orbit behind the comet (Davies and Lovell, 1955; Wu and Williams, 1995b). Also, Perseid outbursts were detected prior to the comet, but with the Earth inside of the comet orbit. In this case, the observed activity behavior before and after the comet is almost symmetric, with the peak activity decreasing by a factor of 10 over a period of 2.8 years in front of, and 4.4 years behind the comet (Jenniskens et al., 1998).

The influence of radiation forces is thought to be strongly particle-size dependent. Kresák (1976) predicted that the effective gravitational field of small and large grains would be different, thus causing the smaller grains to systematically lag the comet more than the large grains. This feature stands out in recent meteoroid stream models. For example, Brown and Jones (1996) noticed that strong activity of faint meteors would be expected in the years after the activity of bright meteors if ejection is in a narrow cone in sunward direction.

Recent Leonid outbursts seem to confirm this hypothesis, with a particle size distribution much less steep in the years prior to perihelion passage than during past post-perihelion Leonid storms. However, that conclusion is probably incorrect. Pre and post-perihelion observations do not represent a continuous variation of dust properties. In fact, during the 1965 return two separate dust components were detected: one filament rich in bright meteors and a sheet rich in faint meteors (Jenniskens, 1996a; Brown et al., 1997). Filament and sheet have a different width, particle size distribution and peak activity variation. Similarly, the Perseid Filament does not show the expected variation of the particle size distribution before and after the comet passed Earth (Jenniskens et al., 1998).

In conclusion, it is likely that radiation forces play a role in shaping the meteoroid streams at early stages of evolution. However, a good understanding of this phase is presently limited by a lack of understanding of the epoch of ejection of the dust seen in meteor outbursts (Section 6) and the relationship of $\beta$ and mass (which may change over time).

\section{In the Plane of the Comet Orbit}

The relationship between cometary dust trails and the streams observed as meteor outbursts at Earth is still by no means clear (Steel, 1994). The main difficulty comes from the extremely narrow width of trails $(\sim 0.001 \mathrm{AU})$ and the typical large distance between comet orbit and Earth's orbit during their encounter (typically up to $0.01 \mathrm{AU}$, sometimes as large as $0.12 \mathrm{AU}$ ). Two classes of mechanisms have been proposed to increase the encounter probability. Either: 1) there is a significant dispersion of matter in the plane of the comet orbit, or 2) the dust trails do not remain at constant distance from the Earth's orbit. A wide variety of scenarios have been proposed in the past, but which of these cases applies to particular situations has been an outstanding problem.

\subsection{Near-comet type: dispersion in the plane of the comet orbit}

The gradual increase and decrease of rates during subsequent Perseid outbursts was thought to be due to wagging dust trails by Wu and Williams (1993). Jenniskens et al. (1998) pointed out that the expected variation of the time of maximum (intimately linked to the relative motion of the dust trail) was opposite to that observed.

Instead, a significant dispersion in the plane of the cometary orbit is necessary. Hence, we speak of "sheets" ("filaments" or "ribbons") instead of "trails". The signature of this is that the duration of the Draconids (Davies and Lovell, 1955) and Perseid outbursts (Jenniskens, 1995a; Jenniskens et al., 1998) does not vary much with a different minimum distance between the comet and Earth orbit. This is also apparent for the Leonid dust sheet, where the minimum distances between comet and Earth's orbit has varied from 0.0013 to $0.0066 \mathrm{AU}$ with no apparent effect on the storm duration (Jenniskens, 1995a). Similarly, the Leonid blanket (the broad dust component responsible for the 196165 and 1994-97 outbursts) had the same thickness during the very different 1965 and 1998 returns (Jenniskens, 1996a). Hence, a general feature of near-comet type outbursts appears to be this significant spread of matter in the plane of the parent comet.

Dispersion of this kind has been found in models of the Perseid shower (Wu and Williams, 1993), although the extent of that dispersion is often related to width variations. Such relation is not observed. More convincing is the dispersion in the model of the Draconid shower $\mathrm{CWu}$ and Williams, 1995a), where the dust distribution reflects the rapid orbital evolution of the parent comet and meteoroids. The dispersion occurs rapidly in discreet events, probably due to close encounters with Jupiter. Only the most recent ejecta would be concentrated close enough to the comet to produce the meteor storms of Draconids in 1933 and 1946. That is consistent with the quite different orbits found for the 1946 and 1953 events: while the 1946 radiant was close to the predicted radiant of 21P/Giacobini-Zinner, the 1953 radiant was 9 degrees off, implicating planetary perturbations. However, the current models predict multiple maxima of prior returns, which are not observed. This forced $\mathrm{Wu}$ and Williams (1995a) to propose that the Draconid meteoroids have a very short lifetime, only tens of years, for which there is no further evidence.

Hence, an outstanding problem remains what is responsible for this dispersion in the plane of the comet orbit and how much of the other mechanism of wagging dust trails contributes to the apparent dispersion.

\subsection{Far-comet type: wagging dust trails}

Until recently, the most common hypothesis for the cause of far-comet type outbursts has been a variant of some form of mass segregation (Lindblad and Porubcan, 1992; Porubcan and Stohl, 1992; Rendtel et al., 1996) or invoked mass concentrations due to orbital resonance with Jupiter (Guth, 1947; Emel'yanenko, 1991). In an alternative hypothesis, dating back to the early recognition that there must be dust trails, it was realized that planets will perturb parts of dust trails differently, resulting in a relative motion of the trail 
with respect to the Earth's path (Plavec, 1955; Kresák, 1958). Lewin (1961) spoke of "Windungen". Typically, the effect of relatively close encounters with the planets was considered to be important. Indeed, Guth (1947) found that Lyrid outbursts occurred when Jupiter and Saturn were near the node of the shower. However, predictions of a 12-year recurrence of the Lyrid outbursts have not been confirmed. In 1994 and 1995, I tried to detect such outburst 12 years after the 1982 event, but without success.

After the 1994 Aurigid outburst established a pattern, I found that the return of far-comet type outbursts of Aurigids (from $\mathrm{C} / \mathrm{Kiess}$ ), alpha-Monocerotids (unknown parent) and Lyrids ( $\mathrm{C} /$ Thatcher) correlated with the position of the planets (Jenniskens, 1997a). For each stream, two planet configurations would lead to an outburst, both of which corresponded to about the same Sun's reflex motion. Clearly, the influence of the combined gravitational field of the planets is important, rather than close encounters with the planets. I recognized such pattern in a model of the Perseid stream by $\mathrm{Wu}$ and Williams (1993) and it has been found also in models of the Leonid and Lyrid streams (Wu and Williams, 1996; Arter and Williams, 1997). It seems as if the meteoroids are moving around the center of mass of the Sun, while the Earth follows a course around the barycenter of the solar system.

From the pattern of large planet positions during prior outbursts, I made a prediction for the return of the alphaMonocerotids in 1995 (Jenniskens, 1995b), which was observed much as predicted. The meteoroids were found to have a long orbital period (Jenniskens et al., 1997a). This enabled us to exclude the hypothesis, reiterated by Rendtel et al. (1996), that a cloud of dust with a 10-year orbital period was responsible. Instead, the return of the shower gave support to the hypothesis that a trail of dust is moving in and out of the Earth's orbit.

The alpha-Monocerotid outburst provided the first detection of a dust trail in the orbit of a long-period comet. As expected, inferred dust densities were 3-5 orders of magnitude lower than derived from the dust trails of short period comets (Sykes and Walker, 1992). The observations provided the first measurement of the particle size distribution in fresh comet ejecta of a long-period comet, showing a surprisingly flat distribution with a cut-off at high masses. That cut-off was interpreted as being the result of a selective ejection of matter in bound orbits (Jenniskens et al., 1997a). The effect has not yet been reproduced in numerical models.

\subsection{Dispersion by wagging motion}

One outstanding problem in studies of the duration of meteor outbursts are the presence of backgrounds in the outburst profiles and some predominance of durations of around one day (Jenniskens, 1995a).

A possible explanation for that has been given in Jenniskens (1997a, b). In time, the wagging motion in combination with the motion of particles along the length of the trail will cause a dispersion of the shower into a characteristic width. A random dispersion over \pm 0.010 AU (all planets) would result in an effective duration of about 0.8 degrees, while Jupiter's influence alone would result in a dispersion of about 0.4 degrees. The time scale of this dispersion is linked to the dispersion along the comet orbit. Models by $\mathrm{Wu}$ and
Williams (1993) do show that Halley-type comets have trail wagging motions at Earth's orbit, which must be masked by the in-plane dispersion.

The proposed scenario for dispersion into a 1-day wide meteor outburst component may have been simulated in a recent model of the Lyrid shower by Arter and Williams (1997). They found that the wagging motion was generated in their model for the Lyrid stream as soon as a sufficient number of meteoroids had completed one orbit. Basically describing a wagging trail, Arter and Williams found their Lyrid stream to be a set of orbits that lay on an elliptical corkscrew, the shape of which is caused by Jovian perturbations. They also found that the structure survives only until the meteoroids complete a further orbit and is then lost because it is being masked by the positions of older and younger meteoroids. This short lifetime of the corkscrew shape of the trail is surprising in the light of previous remarks. It is unlikely that the dispersion occurs as rapidly as in this model.

\section{Changes in Particle Density, Morphology, and Size Distribution}

Observations of meteor outbursts provide unique information about the particle size distribution, the particle's surface-to-mass ratio (on which $\beta$ depends), and the occurrence of fragmentation. Of special interest is whether these features contain information about the epoch of ejection. Outstanding problems are: 1) how do particle morphologies change over time; 2) how does the particle composition change; and 3) how is the particle size distribution affected?

Typically, the particles measured in outbursts are more fragile, more fluffy and have a lower density than annual stream meteoroids. For a review of meteoroid properties deduced from annual stream meteors, see Hughes (1978). Draconids photographed during the meteor storm in 1946 were found to have unusually short trajectories and a high end height (Jacchia et al., 1950). The two most fragile meteoroids measured in the routine Harvard Meteor Project orbit survey are two Draconids from an outburst in 1953. In near identical orbits, these particles had a high beginning and end height, while fragmenting so readily that a long and bright wake formed.

Other evidence of fragile particles came from Porubcan (1974), who found a non-Poissonian distribution of time intervals between meteors at the peak of the 1969 Leonid outburst, thought to be due to relatively recent breakup of meteoroids. More recently, Simek reported a high rate of fragmentation in outburst Perseids from radar observations (Simek, 1996).

The picture is not always that simple, however. Jenniskens et al. (1997a) found the alpha-Monocerotids to be strongly decelerated, indicative of a low density, but not unlike annual Orionids and Perseids. Quite unexpectedly, the outburst meteors penetrated much deeper in the atmosphere than Orionids and Perseids of similar entry velocity, which can only be explained with a loss of volatile materials prior to the collision with Earth, perhaps related to the relative small perihelion distance of this stream.

There is no information to my knowledge about compositional variations, such as a loss of volatiles in time. 
Finally, meteor outbursts provide a direct measurement of the particle size distribution in dust trails (Jenniskens et al., 1997a). It is not surprising that meteor outbursts tend to stand out from annual streams by their anomalous particle size distributions. They are either rich in bright meteors, such as recent Perseid and Leonid outbursts, or they are more abundant in faint meteors such as the Draconid storms. A summary of such information can be found in Jenniskens (1995a). These data hold important clues to the dynamics of meteoroid stream formation, but need further study.

\section{Dispersion into an Annual Stream}

Dust trails represent only a relatively short period of time in the process of meteoroid stream evolution. In a next phase of evolution, the dust rather abruptly moves from the trails into a wider dust component that is responsible for the annual stream. Again, sorting out the relevant mechanisms for different type orbits is the issue at hand.

7.1 Orbital resonances and selective removal of grains

The age of cometary dust trails, 3-30 orbital periods, has been inferred from the dispersion along the orbit, with ejection velocities constrained by the width of the trails (Sykes and Walker, 1992). Similarly, the age of the dust structures responsible for meteor outbursts can be estimated from the number of years these outbursts are observed. While IRAS dust trails imply ages of tens of orbits for short period comets, the concentration of dust near the position of Halley-type comets would suggest very recent ejection. For ejection velocities of $30 \mathrm{~m} / \mathrm{s}$, the Perseid outburst meteoroids should have spread in a mere 8 orbits all along the orbit. Similarly, Leonid storms should occur in more than 1 or 2 years each return.

Often, it has been suggested that the ejecta are therefore very recent. However, that does not need to be the case. Williams (1997) proposed in a recent paper that close encounters with Uranus remove grains effectively from two thirds of the Leonid orbit, whereby a 2:5 orbital resonance protects the region near the parent comet at the present time. That condition prevailed for two thousand years but is due to end in $2160 \mathrm{AD}$.

That scenario was generalized to other near-comet type outbursts by Jenniskens et al. (1998) from a proposed distinction between short-period and Halley-type comets by Chambers (1997). Any physical mechanism that signifies the difference between these comets is important for meteoroid stream evolution, because Halley-type comets are known to produce exclusively near-comet type outbursts, while long-period comets produce exclusively far-comet type outbursts.

Halley-type objects spend a third or so of their time in relatively stable orbits, while librating around mean-motion resonances (Chambers, 1997). That is when a dust sheets can build up. Chambers proposed an analytical equation describing the highest possible orbital period that would allow such orbital resonances (typically $1: j$ ) with the major planets due to indirect perturbations by the planets. Comets with longer orbital period tend to reside in unstable librations for only a few revolutions. The transition from stable to unstable librations is thought to be the distinction between Halley-type and long-period comets.
Those librating motions have the effect of protecting the parent comet from encounters with the major planet, which also prevents the dust close to the comet position from being perturbed. Hence, the dust near the comet is less perturbed by close encounters with the planets than the dust further along the comet orbit (Marsden, 1970). Moreover, the presence of librations around orbital resonances causes the rate of dispersal of meteoroid streams to be much less than in a random situation, confining the distribution of eclipticplane crossings to a narrow region (Emel'yanenko and Bailey, 1996). Because of this, Emel'yanenko (1991) thought the Lyrid outbursts to be an example of such dynamical condensation. However, it is my opinion that the Perseids are a more likely case, because the Lyrids qualify as being long-period in Chambers' equation. In that case, the Perseid meteoroids (in the outburst) are expected to evolve into structures with peaks around mean-motion resonances (mainly 1:n with Jupiter). Unfortunately, the measurement accuracy of the meteor velocity does not allow such structure to be observed at present, in spite of claims by $\mathrm{Wu}$ and Williams (1995b) and Harris and Hughes (1995).

In this scenario, the principal mechanism for putting dust from the dust trail into the annual meteoroid stream is that of close encounters with the planets. This is consistent with the observation that meteor outbursts stand out from annual stream activity (Jenniskens, 1995a).

If this model is correct, the age of the dust in near-comet type outbursts of Halley-type objects is determined by the dynamical lifetime of the comet orbit. In the case of longperiod comets, the dynamical lifetime in terms of number of revolutions is shorter than that of Halley-type comets. Hence, the effect of build-up of dust is less and no near-comet type meteor outbursts are expected. Although appealing, this scenario needs confirmation by meteoroid stream models before it can be accepted.

\subsection{Dispersion by the evolution of the parent body}

The fast dispersion around the orbit of short-period comets in combination with a fast evolution of the comet orbit can lead to ribbon-like streams that are spread out sufficiently to be intersected annually. At this point the concept of annual showers and meteor outbursts becomes ill defined.

In my opinion, the Quadrantids are such case. This shower has the shortest duration among annual showers, is a very strong shower, and has an unusual background with a different particle size distribution. In recent photographic work, Jenniskens et al. (1997b) found a small dispersion of radiants for given speed, concluding that the stream can not be more than 500 years old. This argues directly against current views (e.g. Steel, 1994) that the stream is thousands of years old and originates from comet 96P/Machholz 1, now in a very different orbit. In fact, the narrow dispersion of orbits completely changes the picture of earlier less accurate radar observations and excludes an elaborate orbital evolution towards the formation of twin showers (Babadzhanov and Obrubov, 1992).

The Geminids are unusual in somewhat similar way. Gustafson (1989b) traced a handful of Geminid orbits back to their parent body, 3200 Phaeton, to find that the age of this stream may be only 600-2000 years old. The Geminds is a dense stream and unusual because of the clear mass segre- 
gation along the node. However, that unusual nodal mass segregation may result from the unusual intersection point of Earth with the stream, which is relatively far from perihelion.

\subsection{Dispersion into a background of annual shower activity}

A somewhat similar situation may be the creation of background components to the activity curve of meteoroid streams of Halley type comets. Such background is most apparent in the Perseid stream activity curve, but is also present in the curve of the annual Leonids (Jenniskens, 1994). Harris et al. (1995) attributed this feature to the orbital evolution of the parent body. A model stream profile was generated that matched the observed dispersion of the Perseid shower, providing a 3-D picture of the annual stream. Typically, the node of the orbit will retrogress if the motion is direct, and will advance if the motion is retrograde (Lovell, 1954). The proposed explanation is consistent with the unusual and highly characteristic asymmetry in the background flux profile that was pointed out by Jenniskens (1994).

The precession of the node, spread in inclination, and dispersion of the argument of perihelion by secular perturbations will ultimately lead to the creation of twin streams (c.f. Steel, 1994). Such twin streams are, for example, the north and south branches of the Taurid shower. Many less obvious sets of meteor showers have been proposed to be twin showers (e.g. Babadzhanov and Obrubov, 1992), but it remains an open question if meteoroids typically evolve to this point before being dispersed into the zodiacal cloud.

\section{Dispersion into a Sporadic Background}

At all stages of meteoroid stream evolution, the stream signature may be completely lost in close encounters with the planets, after which the particles no longer are recognized as being part of a meteoroid stream. This is thought to be the dominant mechanism for dispersion into a sporadic background in the case of the Jupiter family of streams with orbital period less than 20 years. The dispersion time scale is estimated to be of order $10^{4}$ years (Olsson-Steel, 1986). An alternative mechanism is the limiting physical lifetime of meteoroids due to impacts with zodiacal dust grains. The time scale for that is of order $10^{4-6}$ years (Grün et al., 1985; Olsson-Steel, 1986). Hence, this mechanism can also remove significant fractions of meteoroids from streams (Steel, 1994).

Multiple encounters with the planets are usually necessary to put the particles from the annual stream in the sporadic background. Hence, most particles follow a route through the annual stream phase. Indeed, most mass of meteoroid streams is found in the annual stream component, not in the outburst component. The latter accounts for only about 10 typical periods of mass loss, while the annual component is typically a factor of 100 more massive (Jenniskens, 1995a). That ratio most likely reflects the relative time scales of dispersion.

Poynting-Robertson drag time scales are usually longer, of order $10^{3-5} a(\mu \mathrm{m})$ years, or $10^{6-9}$ for $1 \mathrm{~mm}-1 \mathrm{~cm}$ grains (Lovell, 1954; Steel, 1994). While both the P-R drag, resulting from the interaction of the Sun's light with a grain in orbital motion, and the Yarkovsky-Radzievskii effect, due to interaction of the Sun's light with a spinning grain, can lead to mass segregation, these mechanisms have been overrated in explaining various aspects of meteoroid streams. Indeed, I find no clear evidence for P-R drag related features of meteoroid streams, in spite of various claims. Perhaps, mainly because of a generally unfavorable encounter geometry, with the Earth crossing the stream perpendicular to the predicted dispersion direction. Of course, P-R drag is expected to play a role in the subsequent dynamical evolution of the cometary grains in the zodiacal cloud (Fig. 5).

\section{Future Work}

Studies of the early stages of meteoroid stream evolution should aim to gradually bridge the gap with studies of cometary dust trails and tails. Although many showers produce meteor outbursts, in the near future the most promising results are expected from the Leonid shower with its possible meteor storms in the years from 1998 until 2000.

Future observations of cometary dust trails should aim to address the features observed in observations of meteor outbursts. In particular, dust trail studies of comet 55P/ Tempel-Tuttle with ISO could address the salient features of the Leonid outbursts. For example, the width of the dust trail should be well described by the flux profile of Leonid storms (effective duration of 0.7 hours). The infrared spectrum should be consistent with a particle size distribution relatively rich in faint meteors (with differential mass distribution index $s=2.2$ ). This narrow component should be strong also in front of the comet and may have a "background" in a lognormal plot of dust density, with a width being a factor of 5 higher. Underlying the narrow component, there should be a broad component rich in large grains with a width 30 times larger. The infrared spectrum of this component should be consistent with a particle size distribution of $s=1.5$. The broad component, if any, should be present before and after the comet position. Behind the comet, I expect the flux scale measured at $1 \mathrm{AU}$ to decay by a factor of 10 every 0.6 years. There should be some variation of the position of the peak flux in the profile due to planetary perturbations. The emission spectrum should remain representative for dust densities with $s=2.2$. The flux profile should remain relatively sharply peaked, with no clear sign of particle size variations across the profile. And studies of such dust trails should attempt to look for dispersion in the cometary plane, by selecting favorable viewing geometries. All of these potential observations will need an adequate explanation.

Clearly, observational and theoretical studies of meteor showers can add a wealth of detail to studies of cometary dust trails and, more general, to studies of the dynamical evolution of cometary ejecta into zodiacal dust.

Acknowledgments. This work benefited greatly from discussions with David Lien. The work was made possible by a grant from NASA’s Planetary Astronomy Program.

\section{References}

Arter, T. R. and I. P. Williams, Periodic behaviour of the April Lyrids, Mon. Not. R. Astron. Soc., 286, 163-172, 1997.

Babadzhanov, P. B. and Yu. V. Obrubov, P/Machholz 1986 VIII and Quadantid meteoroid stream. Orbital evolution and relationship, in 
Asteroids, Comets, Meteors 1991, edited by A. W. Harris and E. Bowell, pp. 27-32, Lunar and Planetary Inst., Houston, 1992.

Betlem, H., C. R. ter Kuile, J. van 't Leven, M. de Lignie, L. Ramon Bellot, M. Koop, C. Angelos, M. Wilson, and P. Jenniskens, Precisely reduced meteoroid trajectories and orbits from the 1995 Leonid meteor outburst, Planet. Space Sci., 45, 853-856, 1997.

Brown, P. and J. Jones, Dynamics of the Leonid meteoroid stream: a numerical approach, in Physics, Chemistry and Dynamics of Inter planetary Dust. ASP Conf. Ser. 104, edited by B. Å. S. Gustafson and M. S. Hanner, pp. 113-116, ASP, San Francisco, 1996.

Brown, P. and J. Rendtel, The Perseid meteoroid stream: Characterization of recent activity from visual observations, Icarus, 124, 414-428, 1996

Brown, P., M. Simek, and J. Jones, Radar observations of the Leonids: 1964-1995, Astron. Astrophys., 322, 687-695, 1997.

Chambers, J. E., Why Halley-types resonate but long-period comets don't: a dynamical distinction between short and long-period comets, Icarus, 125, 32-38, 1997.

Chifo, J. F., The correct evaluation of the sublimation rate of dusty ices under solar illumination, and its implication on the properties of $\mathrm{P} /$ Halley nucleus, Icarus, 130, 549-551, 1997.

Davies, J. G. and A. C. B. Lovell, The Giacobinid meteoroid stream, Mon. Not. R. Astron. Soc., 115, 23-31, 1955.

Davies, J. K., S. F. Green, B. C. Stewart, A. J. Meadows, and H. H. Aumann, The IRAS fast-moving object search, Nature, 309, 315-319, 1984.

Dermott, S. F., D. D. Durda, B. Å. S. Gustafson, S. Jayaraman, J. C. Liou, and Y. L. Xu, Zodiacal dust bands, in Asteroids, Comets Meteors 1993, edited by A. Milani et al., pp. 127-142, 1994.

Emel'yanenko, V. V., Dynamics of the Lyrid meteor swarm, Astron. Vestn., 24, 308-313; Sol. Sys. Res., 24, 199-202, 1991.

Emel'yanenko, V. V. and M. E. Bailey, Regular and stochastic motion of meteoroid streams in Halley-type orbits, in Physics, Chemistry and Dynamics of Interplanetary Dust, ASP Conference Series, Vol. 104 edited by B. Å. S. Gustafson and M. S. Hanner, pp. 121-124, 1996.

Grün, E., H. A. Zook, H. Fechtig, and R. H. Giese, Collisional balance of the meteoritic complex, Icarus, 62, 244-272, 1985.

Gustafson, B. Å. S., Comet ejection and dynamics of nonspherical dust particles and meteoroids, Astrophys. J., 337, 945, 1989a.

Gustafson, B. A. S., Geminid meteoroids traced to cometary activity on Phaethon, Astron. Astrophys., 225, 533-540, 1989b.

Guth, V., On the periodicity of Lyrids, Bull. Astron. Inst. Czech., 1, 1-4, 1947.

Hainaut, O. R., K. J. Meech, H. Boehnhardt, and R. M. West, Early recovery of comet 55P/Tempel-Tuttle, Astron. Astrophys., 1998 (in press).

Harmon, J. K., S. J. Ostro, L. A. M. Benner, K. D. Rosema, R. F. Jurgens, R. Winkler, D. K. Yeomans, D. Choate, R. Cormier, J. D. Giorgini, D L. Mitchell, P. W. Chodas, R. Rose, D. Kelley, M. A. Slade, and M. L. Thomas, Radar detection of the nucleus and coma of comet Hyakutake (C/1996 B2), Science, 278, 1921-1924, 1997.

Harris, N. W. and D. W. Hughes, Perseid meteoroids-the relationship between mass and orbital semimajor axis, Mon. Not. R. Astron. Soc., 273, 992-998, 1995 .

Harris, N. W., K. K. Yau, and D. W. Hughes, The true extent of the nodal distribution of the Perseid meteoroid stream, Mon. Not. R. Astron. Soc., 273, 999-1015, 1995

Harris, W. M., M. R. Combi, R. K. Honeycutt, B. E. Mueller, and F. Scherb, Evidence for interacting gas flow and an extended volatile source distribution in the coma of comet C/1996 B2 (Hyakutake), Science, 277, 676-681, 1997

Hughes, D. W., Meteors, in Cosmic Dust. Chapt. 3, edited by J. A. M. McDonnell, pp. 123-185, John Wiley \& Sons, New York, 1978.

Jacchia, L. G., Z. Kopal, and P. M. Millman, A photographic study of the Draconid meteor shower of 1946, Astrophys. J., 111, 104-133, 1950.

Jenniskens, P., Meteoroid stream activity. I. The annual streams, Astron. Astrophys., 287, 990-1013, 1994

Jenniskens, P., Meteoroid stream activity. II. Meteor outbursts, Astron. Astrophys., 295, 206-235, 1995a

Jenniskens, P., Good prospects for $\alpha$-Monocerotid outburst in 1995, WGN, the Journal of IMO, 23, 84-86, 1995 b.

Jenniskens, P., Meteoroid stream activity. III. Measurements of the first in a new series of Leonid outburst, Meteorics and Planetary Science,
31, 177-184, 1996a.

Jenniskens, P., The first in a new series of Leonid outbursts, in Physics, Chemistry, and Dynamics of Interplanetary Dust, edited by B. A. S. Gustafson and M. S. Hanner, ASP Conf. Ser., 104, 117-120, 1996b.

Jenniskens, P., Meteoroid stream activity. IV. Meteor outbursts and the reflex motion of the Sun, Astron. Astrophys., 317, 953-961, 1997.

Jenniskens, P. and G. Docters van Leeuwen, The $\alpha$-Monocerotids meteor outburst: the cross section of a comet dust trail, Planet. Space Sci., $\mathbf{4 5}$, 1649-1652, 1997.

Jenniskens, P., H. Betlem, M. de Lignie, and M. Langbroek, The detection of a dust trail in the orbit of an Earth-threatening long-period comet, Astrophys. J., 479, 441-447, 1997a.

Jenniskens, P., H. Betlem, M. de Lignie, M. Langbroek, and M. van Vliet, Meteor stream activity. V. The quadrantids, a very young stream, Astron. Astrophys., 327, 1242-1252, $1997 \mathrm{~b}$.

Jenniskens, P., H. Betlem, M. de Lignie, C. ter Kuile, M. C. A. van Vliet, J. van 't Leven, M. Koop, E. Morales, T. Rice, and M. Fulle, On the unusual activity of the Perseid meteor shower (1989-1996) and the dust trail of comet 109P/Swift-Tuttle, Mon. Not. R. Astron. Soc., 1998 (in press).

Jones, J., The ejection of meteoroids from comets, Mon. Not. R. Astron. Soc., 275, 773-780, 1995.

Jones, J. and P. Brown, Modelling the orbital evolution of the Perseid meteoroids, in Physics, Chemistry, and Dynamics of Interplanetary Dust, edited by B. Å. S. Gustafson and M. S. Hanner, ASP Conf. Ser., 104, 105-108, 1997.

Kresák, L., The meteor showers of November 21, 1925 and 1935, and their connexion with comet 1944 I, Bull. Astron. Inst. Czech., 9, 88-96, 1958

Kresák, L., Orbital evolution of the dust streams released from comets, Bull. Astron. Inst. Czech., 27, 35-46, 1976.

Kresák, L., Sources of interplanetary dust, in Solid Particles in the Solar System, IAU Symp. 90, edited by I. Halliday and B. A. McIntosh, pp. 211-222, Reidel, Dordrecht, 1980

Kresák, L., On the ejection and dispersion velocities of meteor particles, Contr. Astron. Obs. Skalnaté Pleso, 22, 123-130, 1992.

Kresák, L., Cometary dust trails and meteor storms, Astron. Astrophys., 279, 646-660, 1993.

Kresák, L. and V. Porubcan, The dispersion of meteors in meteoroid streams. I. The size of the radiant area, BAC, 21, 153-170, 1970.

Kresák, L. and J. Slancikova, On the structure of the Giacobinid meteor shower, Bull. Astron. Inst. Czech., 26, 327-342, 1975.

de Lignie, M. and H. Betlem, Simultane Videometeoren van de Leonidenaktie 1995. Radiant, the Journal of DMS, 68-75, 1997.

Lewin, B. J., Physikalische Theorie der Meteore und die Meteoritische Substanz im Sonnensystem. Teil II, 330 pp., Akademie Verlag, Berlin, 1961.

Lindblad, B. A. and V. Porubcan, Activity of the Lyrid meteoroid stream, in Asteroids, Comets, Meteors 1991, edited by A. W. Harris and E. Bowell, pp. 367-370, Lundar and Planetary Institute, Houston, 1992.

Lindblad, B. A., V. Porubcan, and J. Stohl, The orbit and mean radiant motion of the Leonid meteoroid stream, in Meteoroids and Their Parent Bodies, edited by J. Stohl and I. P. Williams, pp. 177-180, Astron. Inst., Slovak Acad. Sci., Bratislava, 1993.

Lovell, A. C. B., Meteor Astronomy, 463 pp., Clarendon Press, Oxford, 1954.

Mann, I., The contribution of asteroid dust to the interplanetary dust cloud: The impact of ULYSSES results on the understanding of dust production in the asteroid belt and of the formation of the IRAS dust bands, Icarus, 120, 399-407, 1996.

Marsden, B. G., On the relationship between comets and minor planets, Astron. J., 75, 206-217, 1970.

Novikov, G. G., P. Pecina, and N. A. Konovalova, Bulk densities of meteoroids, Astron. Astrophys., 360, 991-994, 1996.

O'Ceallaigh, D. P., A. Fitzsimmons, and I. P. Williams, CCD photometry of comet 109P/Swift-Tuttle, Astron. Astrophys., 297, L17-L20, 1995.

Olsson-Steel, D., The origin of the sporadic meteoroid component, Mon. Not. R. Astron. Soc., 219, 47-73, 1986.

Pittich, E. M., in Dynamika Malych Cial Ukladu Sloneznego, edited by K. Ziolkowski, pp. 55, PAN, Warszawa, 1991 (see Kresák, 1992).

Plavec, M., Meteor strams at early stages of evolution, in Meteors, Special Supplement (Vol. 2), J. Atmos. Terr. Phys., pp. 168-177, 1955. 
Porubcan, V., On the structure of the 1969 Leonid meteor shower, Bull. Astron. Inst. Czech., 25, 353-361, 1974.

Porubcan, V. and J. Stohl, Bust of the 1969 Leonids and 1982 Lyrids, in Asteroids, Comets, Meteors 1991, edited by A. W. Harris and E. Bowell, pp. 469-472, Lundar and Planetary Inst., Houston, 1992.

Rendtel, J., P. Brown, and S. Molau, The 1995 outburst and possible origin of the alpha-Monocerotid meteoroid stream, Mon. Not. R. Astron. Soc., 279, L31-L36, 1996.

Sekanina, Z., Meteoric storms and formation of meteoroid streams, in Asteroids, Comets, Meteoric Matter, edited by C. Cristesu, W. J. Klepczynski, and B. Millet, pp. 239-267, 1975.

Simek, M., Fine structure of the 1985 Giacobinids, Astron. Astrophys., 284 276-280, 1994.

Simek, M., Fragmenting particles during 1989-1994 Perseid radar observations, in Physics, Chemistry, and Dynamics of Interplanetary Dust, edited by B. Å. S. Gustafson and M. S. Hanner, ASP Conf. Ser., 104, 79-82, 1996.

Simek, M. and P. Pecina, Activity of the new filament in the Perseid meteoroid stream, in Physics, Chemistry, and Dynamics of Interplanetary Dust, edited by B. A. S. Gustafson and M. S. Hanner, ASP Conf. Ser., 104, 109-112, 1996.

Steel, D., Meteoroid streams, in Asteroids, Comets, Meteors 1993, edited by A. Milani et al., pp. 111-126, 1994.

Sykes, M. V. and R. G. Walker, Cometary dust trails. I. Survey, Icarus, 95, 180-210, 1992.

Sykes, M. V., L. A. Lebofsky, D. M. Hunten, and F. Low, The discovery of dust trails in the orbits of periodic comets, Science, 232, 1115-1117, 1986.

Sykes, M. V., D. J. Lien, and R. G. Walker, The Tempel 2 dust trail, Icarus, 86, 236-247, 1990.
Watanabe, J.-I., T. Nakamura, M. Tsutsumi, and T. Tsuda, Radar observations of the strong activity of a Perseid meteor shower in 1991, Publ. Astron. Soc. Japan, 44, 677-685, 1992.

Whipple, F. L., A comet model. II. Physical relations for comets and meteors, Astrophys. J., 113, 464-474, 1951.

Williams, I. P., The dynamics of meteoroid streams, in Meteoroids and Their Parent Bodies, edited by J. Stohl and I. P. Williams, pp. 31-40, Astron. Inst. Slovak Acad. Sci., Bratislava, 1993.

Williams, I. P., The evolution of meteoroid streams, in Physics, Chemistry, and Dynamics of Interplanetary Dust, edited by B. A. S. Gustafson and M. S. Hanner, ASP Conf. Ser., 104, 89-99, 1996a.

Williams, I. P., What can meteoroid streams tell us about the ejection velocities of dust from comets?, Earth, Moon and Planets, 72, 321-326, 1996 b.

Williams, I. P., The Leonid meteor shower: why are there storms but no regular annual activity?, Mon. Not. R. Astron. Soc., 292, L37-L40, 1997.

Williams, I. P. and Z. Wu, The current Perseid meteor shower, Mon. Not. R. Astron. Soc., 269, 524-528, 1994.

$\mathrm{Wu}, \mathrm{Z}$. and I. P. Williams, The Perseid meteor shower at the current time, Mon. Not. R. Astron. Soc., 264, 980-990, 1993.

Wu, Z. and I. P. Williams, P/Giacobini-Zinner and the Draconid meteor shower, Planet. Space Sci., 43, 723-731, 1995a.

$\mathrm{Wu}, \mathrm{Z}$. and I. P. Williams, Gaps in the distribution of semimajor axes of the Perseid meteors, Mon. Not. R. Astron. Soc., 276, 1017-1023, 1995b.

Wu, Z. and I. P. Williams, Leonid meteor storms, Mon. Not. R. Astron. Soc., 280, 1210-1218, 1996

Yeomans, D. K., Comet Tempel-Tuttle and the Leonid meteors, Icarus, 47, 492-499, 1981.

P. Jenniskens (e-mail: peter@max.arc.nasa.gov) 